\title{
Acute kidney injury following the first stage of the ALPPS procedure: A case report
}

\author{
WEIJIE TAO, XIAOJU SHI and GUANGYI WANG \\ Department of Hepatobiliary Surgery, The First Hospital of Jilin University, Changchun, Jilin 130021, P.R. China
}

Received March 24, 2016; Accepted March 3, 2017

DOI: 10.3892/etm.2018.5789

\begin{abstract}
Associating liver partition and portal vein ligation for staged hepatectomy (ALPPS) is a novel approach for performing liver resection, and the number of clinical applications of this technique has rapidly increased within recent years. ALPPS is important in patients who have insufficient residual liver volume and cannot undergo radical hepatic resection. The most common postoperative complications of ALPPS include biliary fistula and infection. To date, postoperative acute kidney injury following ALPPS has not been reported. The current study reports the case of a 63 -year-old patient with hepatitis B-induced cirrhosis who underwent the first stage of ALPPS without completion of the second step. The patient developed postoperative acute kidney injury following ALPPS. The present case study suggests that the use of ALPPS in patients at risk of chronic renal damage should be approached with caution in order to avoid postoperative acute kidney injury. Furthermore, improvements in surgical techniques and skills of the surgeons performing the procedure are required to reduce the surgery duration and improve patient outcomes.
\end{abstract}

\section{Introduction}

Associating liver partition and portal vein ligation for staged hepatectomy (ALPPS) is a novel strategy for liver resection that was originally established in 2007 by the German surgeon Hans Schlitt (1,2), and was first reported in 2012 by Schnitzbauer et al (3). The original method utilizing laparotomy surgery has been improved by a completely laparoscopic surgery (4). However, the potential postoperative complications and mortality subsequent to ALPPS remains high, and limited long-term follow-up data exists to date. The most common postoperative complications of ALPPS include biliary fistula (10.2-24\%) and infection (7.7-20\%) (5). To the best of our knowledge, postoperative acute kidney injury

Correspondence to: Dr Guangyi Wang, Department of Hepatobiliary Surgery, The First Hospital of Jilin University, 71 Xinmin Street, Changchun, Jilin 130021, P.R. China

E-mail:wgymd@sina.com

Key words: liver cancer, ALPPS, complication, acute kidney injury following ALPPS in patients with posthepatitic cirrhosis has not yet been reported.

The present study reported the case of a patient with hepatitis B-induced cirrhosis who experienced postoperative acute kidney injury after the first stage of the ALPPS procedure. The current case report aimed to analyze the potential causes of this complication and suggests measures that can be employed in clinical practice to prevent postoperative acute kidney injury, thereby improving the patient outcome.

\section{Case report}

In May 2015, a 63-year-old man was admitted to the Department of Hepatobiliary Surgery at the First Hospital of Jilin University (Changchun, China), because of abdominal pain and faintness for 2 months. The patient provided informed consent for selective surgery, and the protocol was approved by the Institutional Review Board of our hospital. The patient's medical history revealed that he suffered from chronic hepatitis B for $>30$ years, diabetes for 7 years (with regular injection of insulin; fasting blood-glucose was controlled under $\sim 7.3 \mathrm{mmol} / \mathrm{l}$ ), and hypertension for 10 years (blood pressure, $150 / 90 \mathrm{mmHg}$ ). There were no positive signs of kidney disease, with blood levels of creatinine of $60.6 \mu \mathrm{mol} / 1$ (normal range, $41-73 \mu \mathrm{mol} / \mathrm{l}$ ) and glomerular filtration rate of $95 \mathrm{ml} / \mathrm{min}$ (normal range, $80-120 \mathrm{ml} / \mathrm{min}$ ) during the physical examination.

Computed tomography examination (Fig. 1A and B) displayed multiple low density nodules of $0.8-6.8 \mathrm{~cm}$ in diameter in the anterior segment of the right lobe of the cirrhotic liver, which presented significantly heterogeneous enhancement at the arterial phase and washout in the portal venous and delayed phases. In addition, ultrasonography (Fig. 1C) revealed a large solid tumor in the right lobe of the liver, with a size of $7.0 \times 5.8 \mathrm{~cm}$

Various laboratory tests were also performed. The results of the liver function test were as follows: Elevated aspartate aminotransferase, $118.0 \mathrm{U} / 1$ (normal range, 0-31 U/1); elevated alanine aminotransferase, 156.1 U/1 (normal range, 0-32 U/1); elevated $\gamma$-glutamyl transpeptidase, $75.9 \mathrm{U} / 1$ (normal range, 7-45 U/l); elevated total bilirubin, $30.4 \mu \mathrm{mol} / 1$ (normal range, $<17.1 \mu \mathrm{mol} / 1$ ); and elevated indirect bilirubin, $22.2 \mu \mathrm{mol} / 1$ (normal range, 0-15 $\mu \mathrm{mol} / \mathrm{l}$ ). In addition, the renal function examination demonstrated normal blood urea nitrogen (BUN) at $2.93 \mathrm{mmol} / 1$ (normal range, 2.86-7.14 $\mu \mathrm{mol} / \mathrm{l}$ ) and normal 

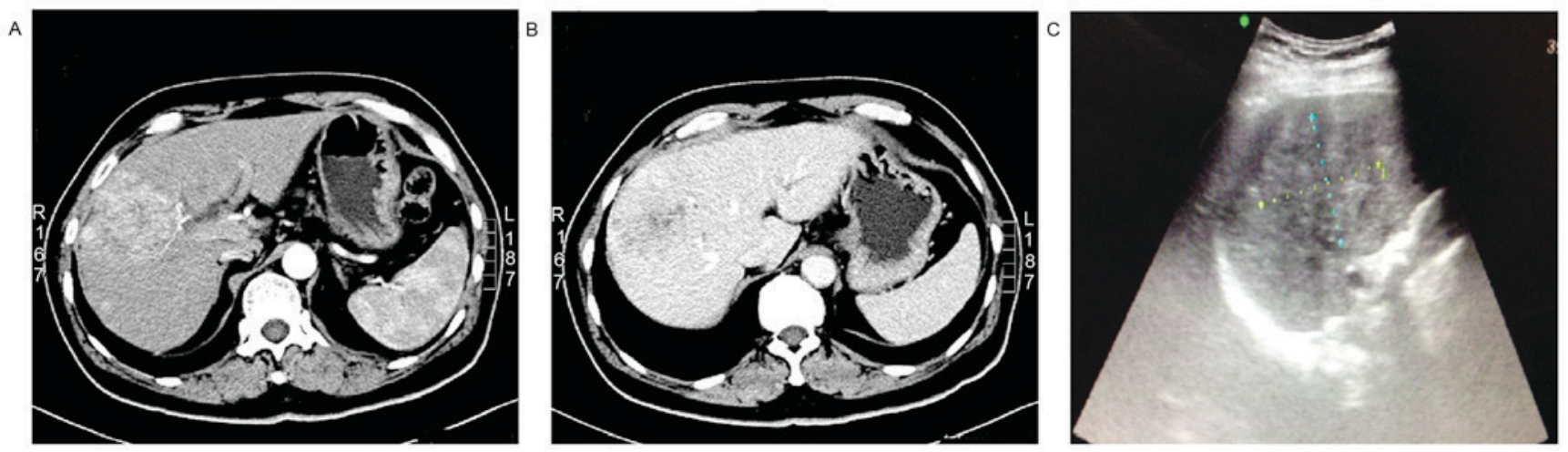

Figure 1. Preoperative liver contrast-enhanced CT and ultrasound imaging in the patient. The contrast-enhanced CT displayed (A) a liver mass at the VIII segment of the right lobe that was significantly enhanced at the hepatic arterial phase, and (B) evident washout in the portal vein phase. (C) Ultrasonography showed a large solid tumor $(7.0 \times 5.8 \mathrm{~cm})$ in the right lobe of the liver. CT, computed tomography.

creatinine level at $60.6 \mu \mathrm{mol} / 1$ (normal range, $41-73 \mu \mathrm{mol} / \mathrm{l}$ ). A routine urine culture was negative for urine protein. Hepatitis $\mathrm{B}$ virus (HBV) quantitative test revealed an elevated level of $5.4 \times 10^{7} \mathrm{IU} / \mathrm{ml}$ (normal range, $<500 \mathrm{IU} / \mathrm{ml}$ ), while the serum alphafetoprotein level was elevated at $25.34 \mathrm{ng} / \mathrm{ml}$ (normal range, $0.89-8.78 \mathrm{ng} / \mathrm{ml}$ ) and carbohydrate antigen was elevated at $19,978.97 \mathrm{U} / \mathrm{ml}$ (normal range, $0-6.9 \mathrm{U} / \mathrm{ml}$ ). The indocyanine green retention rate at $15 \mathrm{~min}$ was normal at $8.4 \%$ (normal range, $<10 \%)$. Furthermore, the preoperative liver volume measurements indicated that the volume of the left lobe in the liver was $520 \mathrm{ml}$, the volume of the left external lobe in the liver was $300 \mathrm{ml}$ and the standard liver volume (SLV) was $1,420 \mathrm{ml}$. These results indicated a dysfunction of the liver.

The preoperative clinical diagnosis was primary liver cancer with decompensated hepatitis B-induced cirrhosis. Entecavir (0.5 mg/day; Sino American Shanghai Squibb Pharmaceuticals Ltd., Shanghai, China) was administered for antiviral treatment following admission. Blood glucose levels under fasting conditions and subsequent to meals were monitored, and the dose of insulin was adjusted accordingly. As the nodules were predominately located in the right lobe of the liver and closely connected to the middle and right hepatic veins, right hemihepatectomy or right hepatictrisegmentectomy was required to achieve an $\mathrm{R} 0$ resection in the patient. However, as the patient suffered from hepatitis B-induced cirrhosis, for which the postoperative future liver remnant size would be $<40 \%$ of the SLV (5) and he could not tolerate one-stage complete resection of the tumor, laparoscopic ALPPS was selected.

Intraoperative probe examination detected moderate cirrhosis of the liver with a firm, pale mass with a diameter of $7 \mathrm{~cm}$ in the right anterior lobe, which was closely connected to the middle and right hepatic veins. In addition, there were several nodules of $0.5-1.5 \mathrm{~cm}$ in diameter in the right posterior lobe and left internal lobe, while another $0.8-\mathrm{cm}$ nodule was located on the surface of the left external lobe of the liver. These lesions are typically observed in patients with primary liver cancer. ALPPS was performed (6), during which the lesion that was $1.5 \mathrm{~cm}$ in diameter in the left external lobe of the liver was locally resected. The right branch of the hepatic vein and the right hepatic artery were ligated, and preset lines were identified for the secondary surgery. Postoperative pathology confirmed the diagnosis of primary hepatocellular carcinoma (HCC) with moderate differentiation.
Postoperatively, the patient's urine output gradually decreased from the first day following surgery, with blood levels of creatinine and BUN continuously increasing. On the third day following ALPPS, the levels of creatinine and BUN in the patient's blood were elevated, at $386.4 \mu \mathrm{mol} / \mathrm{l}$ (normal range, $41-73 \mu \mathrm{mol} / \mathrm{l}$ ) and $24.58 \mathrm{mmol} / 1$ (normal range, $2.86-7.14 \mathrm{mmol} / \mathrm{l})$, respectively, which are considerably higher than the normal values. All these tests (urine output, creatinine and BUN) indicated a decline in kidney function, and therefore, the patient was diagnosed with postoperative acute kidney injury, according to the evaluation criteria for acute kidney injury from the Acute Kidney Injury Work Group (7). Thus, the patient was treated with continuous dialysis for 3 days, beginning on postoperative day 3 , resulting in improvement in the renal function. However, higher blood levels of creatinine $(707.9 \mu \mathrm{mol} / \mathrm{l})$ and BUN $(81.9 \mathrm{mmol} / \mathrm{l})$ were detected in the patient when dialysis was stopped, while even volume expansion and fluid supplement therapy failed to decrease these levels. The patient was recommenced on dialysis for 2 days, beginning on postoperative day 16, and the levels of creatinine and BUN decreased to $136.4 \mu \mathrm{mol} / 1$ (normal range, $41-73 \mu \mathrm{mol} / \mathrm{l}$ ) and $26.44 \mathrm{mmol} / 1$ (normal range, 2.86-7.14 mmol/l), respectively (Fig. 2). Subsequent to symptomatic treatments, such as liver and kidney protection and fluid therapy (reduced glutathione, 1,200 mg/day, i.v.; magnesium isoglycyrrhizinate, $100 \mathrm{mg} /$ day, i.v.; spironolactone, $60 \mathrm{mg} /$ day, orally administered), renal function improved and the 24-h urine volume gradually returned to normal. Other complications were also observed, such as refractory ascites and bacterial peritonitis, although the symptoms of peritonitis were gradually improved after abdominal drainage, hemofiltration and administration of antibiotics (vancomycin, 2 g/day, i.v.) effective against Enterococcus faecium. However, the patient refused further treatment due to financial reasons, and selected to be discharged from hospital care.

The patient attended scheduled follow-up appointments until October 2015. The final results of the liver function test were as follows: Aspartate aminotransferase, $200.0 \mathrm{U} / 1$ (normal range, 0-31 U/1); alanine aminotransferase, $250.5 \mathrm{U} / 1$ (normal range, 0-32 U/1); $\gamma$-glutamyl transpeptidase, $100 \mathrm{U} / 1$ (normal range, 7-45 U/l); total bilirubin, $120.6 \mu \mathrm{mol} / 1$ (normal range, $<17.1 \mu \mathrm{mol} / \mathrm{l})$; and indirect bilirubin, $80.3 \mu \mathrm{mol} / 1$ (normal range, 0-15 $\mu \mathrm{mol} / \mathrm{l}$ ). In addition, the renal function 

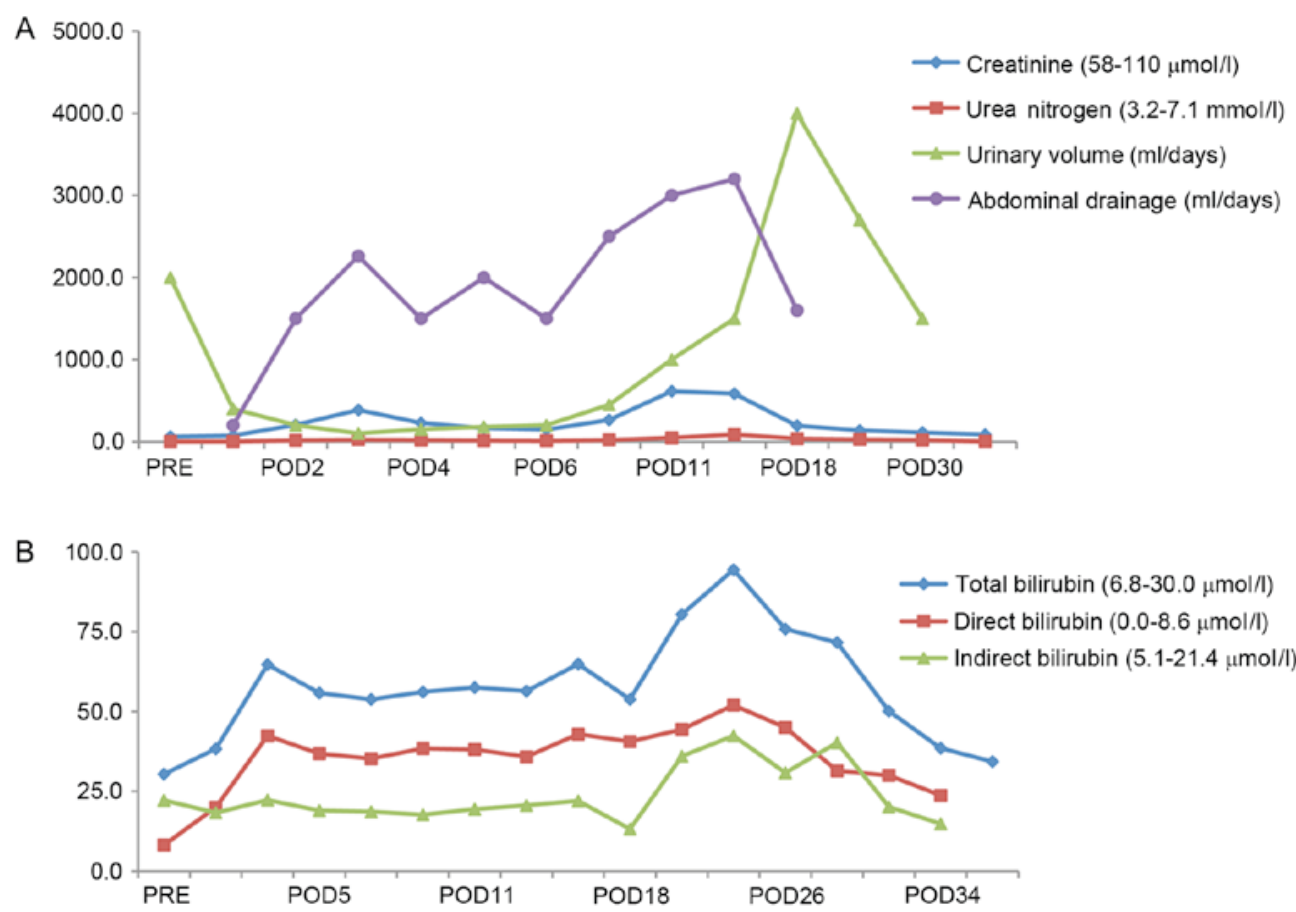

Figure 2. Laboratory tests demonstrated changes in parameters associated with the (A) renal function and (B) liver function of the patient in the preoperative and postoperative periods. Parentheses show the reference ranges and/or units. PRE, preoperative period; POD, postoperative day.

examination demonstrated BUN at $30.23 \mathrm{mmol} / 1$ (normal range, $2.86-7.14 \mu \mathrm{mol} / \mathrm{l})$ and creatinine level at $180.6 \mu \mathrm{mol} / \mathrm{l}$ (normal range, $41-73 \mu \mathrm{mol} / \mathrm{l}$ ). As the patient only underwent the first stage of the ALPSS procedure, the liver tumor was still present. Liver and kidney function appeared to be worse than when he was first admitted.

\section{Discussion}

Patients with multiple intrahepatic tumors or a large liver tumor, regardless of the functional residual liver volume, are usually treated with liver resection. However, concerns have been raised regarding the small liver remnants and the development of postoperative complications in these patients (2). Portal vein embolization (7) and portal vein ligation $(8,9)$ are two conventional techniques, which are widely used prior to liver resection to maintain sufficient liver volumes following surgery. Recently, ALPPS has emerged as a novel procedure to rapidly increase the residual liver volume with a 74-99\% increase in the remnant liver in the short term (9 days). In addition, the ALPPS technique meets the safe liver resection criteria (3), while it reduces the surgery duration and the incidence of postoperative hepatic failure. As of September 2015, 553 patients were registered as having undergone ALPSS from 84 medical centers worldwide (www.alpps.net) (10). However, ALPPS has certain limitations, including high morbidity and mortality rates, and postoperative complications. In the present case report, a patient who underwent the first stage of the ALPSS procedure without completion of the second stage was reported. The patient experienced acute kidney injury following ALPPS, a rare postoperative complication.

To date, a limited number of published case reports exist on postoperative complications following ALPPS, and acute kidney injury has not previously been reported as a postoperative complication of ALPPS. Therefore, there is no predictive guidlines that can be followed in order to avoid postoperative renal damage following ALPPS. Schadde et al (10) analyzed the early postoperative survival rates and safety of the procedure in 202 registered ALPPS patients. In their report, the independent risk factors associated with postoperative complications were as follows: Intraoperative erythrocyte transfusion, surgery duration of the first stage ALPPS of $>300$ min, patient age of $>60$ years, and liver metastasis other than colorectal liver metastasis. In the present case study, the patient was 63 years old with a history of hypertension and diabetes, which are known to induce chronic renal damage. The patient was, therefore, at risk of acute kidney injury (10). Furthermore, the long surgery duration of 380 min during the first stage of ALPPS, along with the adverse effects of anesthesia and surgical stress on renal function possibly increased the risk and contributed to the development of acute kidney injury. Of patients undergoing the ALPPS procedure, $70 \%$ of cases presented liver metastasis from colorectal cancer, $\sim 17 \%$ of patients presented primary liver cancer $(2,10,11)$, and a small number of patients had liver cancer secondary to hepatitis-induced cirrhosis. The latter group of patients are more likely to suffer with viral nephropathy and are at a high risk of postoperative acute kidney injury.

Suggestions of the present study for measures that can be performed in clinical practice in order to reduce the risk of postoperative acute renal injury and improve patient outcomes also merit attention. Firstly, when the postoperative urine volume or renal function are decreased, an insufficient circulating blood volume should be considered. Therefore, the first step is to replenish the circulating volume, and subsequent diuretics should increase the daily urine output with an adequate fluid intake. Secondly, if patients are diagnosed with HBV infection, antiviral drug therapy should be used 
prior to liver resection (12) in order to avoid impairment in kidney function caused by HBV infection. Antibiotics with less adverse effects on renal function should be selected when spontaneous bacterial peritonitis occurs. Abdominal drainage should be performed if refractory ascites are suspected. Finally, if no improvement in renal function is reported or the patient develops metabolic acidosis and severe hyperkalemia, then renal replacement therapy should be considered to remove toxins (13). Hemofiltration therapy can also help to restore liver function. In the present study, the hepatic function of the patient was improved subsequent to the second hemofiltration treatment. Overall, when renal insufficiency occurs following ALPPS, the patient should be treated as soon as possible to avoid further deterioration of potential acute kidney injury or failure.

In conclusion, the use of ALPPS in patients at risk of chronic renal damage should be carefully considered in order to avoid the development of postoperative acute kidney injury following ALPPS. Furthermore, improvement in surgical techniques and skills are required to reduce the surgical duration and improve the clinical outcomes of patients.

\section{References}

1. de Santibañes E and Clavien PA: Playing Play-Doh to prevent postoperative liver failure: The 'ALPPS' approach. Ann Surg 255: 415-417, 2012.

2. Bertens KA, Hawel J, Lung K, Buac S, Pineda-Solis K and Hernandez-Alejandro R: ALPPS: Challenging the concept of unresectability-a systematic review. Int J Surg 13: 280-287, 2015.

3. Schnitzbauer AA, Lang SA, Goessmann H, Nadalin S, Baumgart J, Farkas SA, Fichtner-Feigl S, Lorf T, Goralcyk A, Hörbelt R, et al: Right portal vein ligation combined with in situ splitting induces rapid left lateral liver lobe hypertrophy enabling 2 -staged extended right hepatic resection in small-for-size settings. Ann Surg 255: 405-414, 2012.
4. Machado MA, Makdissi FF and Surjan RC: Totally laparoscopic ALPPS is feasible and may be worthwhile. Ann Surg 256: e13; author reply e16-e19, 2012.

5. Zhang GQ, Zhang ZW, Lau WY and Chen XP: Associating liver partition and portal vein ligation for staged hepatectomy (ALPPS): A new strategy to increase resectability in liver surgery. Int J Surg 12: 437-441, 2014.

6. Torres OJ, Fernandes Ede S, Oliveira CV, Lima CX, Waechter FL, Moraes-Junior JM, Linhares MM, Pinto RD, Herman P and Machado MA: Associating liver partition and portal vein ligation for staged hepatectomy (ALPPS): The Brazilian experience. Arq Bras Cir Dig 26: 40-43, 2013 (In English, Portuguese).

7. Kidney Disease Improving Global Outcomes (KDIGO) Acute Kidney Injury Work Group: KDIGO clinical practice guideline for acute kidney injury. Kidney Inter 2 (Suppl): 1-138, 2012

8. Clavien PA, Petrowsky H, DeOliveira ML and Graf R: Strategies for safer liver surgery and partial liver transplantation. N Engl J Med 356: 1545-1559, 2007.

9. Kianmanesh R, Farges O, Abdalla EK, Sauvanet A, Ruszniewski P and Belghiti J: Right portal vein ligation: A new planned two-step all-surgical approach for complete resection of primary gastrointestinal tumors with multiple bilateral liver metastases. J Am Coll Surg 197: 164-170, 2003.

10. Schadde E, Ardiles V, Robles-Campos R, Malago M, Machado M, Hernandez-Alejandro R, Soubrane O, Schnitzbauer AA, Raptis D, Tschuor C, et al: Early survival and safety of ALPPS: First report of the international ALPPS registry. Ann Surg 260: 829-836; discussion 836-838, 2014.

11. Correa-Gallego C, Berman A, Denis SC, Langdon-Embry L, O'Connor D, Arslan-Carlon V, Kingham TP, D'Angelica MI, Allen PJ, Fong Y, et al: Renal function after low central venous pressure-assisted liver resection: Assessment of 2116 cases. HPB (Oxford) 17: 258-264, 2015

12. Zhang B, Xu D, Wang R, Zhu P, Mei B, Wei G, Xiao H, Zhang B and Chen $X$ : Perioperative antiviral therapy improves safety in patients with hepatitis B related HCC following hepatectomy. Int J Surg 15: 1-5, 2015.

13. Sourianarayanane A, Raina R, Garg G, McCullough AJ and O'Shea RS: Management and outcome in hepatorenal syndrome: Need for renal replacement therapy in non-transplanted patients. Int Urol Nephrol 46: 793-800, 2014. 